\title{
Identificación y técnica de medición de las consolidaciones subpleurales mediante ultrasonido en COVID-19
}

Identification and technique of measuring subpleural consolidations using ultrasound in COVID-19

Tecnica de identificação e medição de consolidações subpleurais por ultrassom no COVID-19

\author{
Karen Itzel González Martínez, ${ }^{*}$ Felipe de Jesús Montelongo, ${ }^{*}$ Roberto Emmanuel Islas Ávila, ${ }^{*}$ David Gatica Castro, ${ }^{\ddagger}$ \\ Diana Vanessa González Martínez§
}

\section{RESUMEN}

En un principio se pensó que el ultrasonido no era útil para el estudio del pulmón al tomar como base el principio ultrasonográfico en el cual el aire refleja las ondas sonoras actuando como una barrera biológica. Comúnmente las imágenes de pulmón en pacientes críticamente enfermos se realiza de manera rutinaria por la toma de radiografía de tórax a la cabecera del paciente (RXT) o la de la tomografía computarizada torácica, la cual, aunque es el estándar de oro para la toma de imágenes de pulmón, es cara y no se puede realizar en una forma rutinaria. Para la realización de la radiografía de tórax implica traslado al departamento de radiología, lo cual pone en riesgo a un paciente crítico, as como la RXT portátil se limita a una pobre calidad de imagen y baja sensibilidad, es aquí donde cobra importancia el ultrasonido pulmonar al ser un método fácil y rápido que puede realizarse a la cabecera del paciente y de menor costo. Objetivo: El objetivo de este estudio es proponer un protocolo mediante una técnica ultrasonográfica para identificar las características de las consolidaciones subpleurales y su importancia en la evolución de la enfermedad en pacientes con diagnóstico de COVID-19 en el departamento de terapia intensiva del Hospital General de Ecatepec.

Material y métodos: Previa autorización del comité de ética se realizó un estudio prospectivo, analítico, en el departamento de terapia intensiva mediante un ultrasonido VINNO 5 y con transductor lineal y convexo se identificaron las consolidaciones subpleurales, se tomaron medidas y se siguió la evolución en los pacientes ingresados a la Unidad de Terapia Intensiva del Hospital Genera de Ecatepec.

Conclusiones: En este estudio se demostró que las consolidaciones subpleurales son cambios presentes en la infección por SARS-CoV-2 y que la identificación y el monitoreo de su tamaño mediante ultrasonido durante el curso clínico de la enfermedad pueden dar pauta a la toma de decisiones e inferir en el diagnóstico y pronóstico de dicha patología.

Palabras clave: Consolidación subpleural, ultrasonido, COVID-19.

\section{ABSTRACT}

At first it was thought that ultrasound was not useful for the study of the lung based on the ultrasound principle in which air reflects sound waves acting as a biological barrier. Commonly, lung imaging in critically ill patients is performed routinely by taking a chest $X$-ray at the bedside (CXR) or a thoracic computed tomography which, although it is the gold standard for imaging of lung is expensive and cannot be performed routinely. To carry out the chest $X$-ray involves transfer to the radiology department which puts a critical patient at risk as well as portable CXR is limited to poor image quality and low sensitivity, this is where pulmonary ultrasound becomes important, as it is an easy, fast method that can be performed at the bedside and at a lower cost.

Objective: The objective of this study is to propose a protocol using an ultrasound technique to identify the characteristics of subpleural consolidations and their importance in the evolution of the disease in patients with a diagnosis of COVID-19 in the Intensive Care department of the General Hospital of Ecatepec.

Material and methods: With prior authorization from the ethics committee, a prospective analytical study was carried out in the Intensive Care departmen

\footnotetext{
* Hospital General de Ecatepec Las Américas.

‡ Hospital General de Zona No. 8, Córdoba, Veracruz.

$\S$ Facultad de Estudios Superiores Zaragoza, UNAM.
}

Recibido: 16/10/2020. Aceptado: 26/10/2020

Citar como: González MKI, Montelongo FJ, Islas ÁRE, Gatica CD, González MDV Identificación y técnica de medición de las consolidaciones subpleurales mediante ultrasonido en COVID-19. Med Crit. 2021;35(4):182-185. https://dx.doi.org/10.35366/101156

www.medigraphic.com/medicinacritica using a VINNO 5 ultrasound and with a linear and convex transducer, subpleural consolidations were identified, measurements were taken and the evolution was followed in hospitalized patients to the Intensive Therapy Unit of the General Hospital of Ecatepec.

Conclusions: In this study, it was demonstrated that subpleural consolidations are changes present in the SARS-CoV-2 infection and that the identification and monitoring of their size by ultrasound during the clinical course of the disease can guide us in the taking of decisions and infer in the diagnosis and prognosis of said pathology.

Keywords: Subpleural consolidation, ultrasound, COVID-19.

\section{RESUMO}

No início, pensava-se que o ultrassom não era útil para o estudo do pulmão, pois era baseado no princípio do ultrassom em que o ar reflete as ondas sonoras atuando como uma barreira biológica. $A$ imagem pulmonar em pacientes criticamente enfermos é comumente realizada em um rotina fazendo uma radiografia de tórax à beira do leito (RXT) ou tomografia computadorizada de tórax, que, embora seja o padrão de ouro para imagens de pulmão, é caro e não pode ser realizado rotineiramente. Para realizar a radiografia de tórax envolve transferência ao departamento de radiologia, o que também coloca em risco um paciente crítico, assim como o RXT portátil é limitado à qualidade de imagem ruim e baixa sensibilidade, é aqui que o ultrassom pulmonar se torna importante como método rápido e fácil que pode ser realizado à beira do leito do paciente e com menor custo.

Objetivo: O objetivo deste estudo é propor um protocolo por meio de um técnica ultrassonográfica para identificar as características das consolidações subpleurais e sua importância na evolução da doença em pacientes com diagnóstico de COVID-19 na unidade de terapia intensiva do Hospital General de Ecatepec.

Material e métodos: Com autorização prévia do comitê de ética, foi realizado estudo prospectivo e analítico em unidade de terapia intensiva utilizando um ultrassom VINNO 5 e com um transdutor linear e convexo identificaram o consolidações subpleurais, foram tomadas medidas e acompanhou-se a evolução dos pacientes internados na Unidade de Terapia Intensiva do Hospital General de Ecatepec.

Conclusões: Neste estudo demonstrou-se que as consolidações subpleurais são alterações presentes na infecção por SARS-CoV-2 e que a identificação e monitoramento de seu tamanho por ultrassom durante o curso clínico da doença podem orientar a tomada de decisão e inferir no diagnóstico e o prognóstico dessa patologia.

Palavras-chave: Consolidação subpleural, ultrassom, COVID-19.

\section{INTRODUCCIÓN}

Es evidente que la ecografía pulmonar tiene múltiples ventajas sobre la tomografía computarizada (TC) y el equipo de rayos $X$. En particular, la sonda de matriz lineal de alta frecuencia de 9-15 $\mathrm{MHz}$ puede mostrar claramente la morfología y los cambios de las lesiones subpleurales y los cambios del contenido de aire y agua en los tejidos peripulmonares consolidados. ${ }^{1}$

Un hallazgo descrito en COVID-19 es la visualización de consolidaciones subpleurales, mismas que pueden ser identificadas a través de la interrupción focal de la línea pleural. Cuando la consolidación es de mayor cuan- 
tía, el parénquima pulmonar puede adquirir un aspecto ecográfico similar al parénquima hepático sano y puede exhibir imágenes hiperecogénicas estáticas o móviles que corresponden al broncograma aéreo descrito en la $\mathrm{TC}^{2}$ Las consolidaciones subpleurales aparecen inicialmente en zonas basales y posteriores para generalizarse a todo el pulmón e ir aumentando de tamaño si sigue progresando el cuadro. Son pequeñas áreas hipoecoicas, de base en la pleura y que se movilizan con la respiración. Presentan un límite profundo irregular, están rodeadas de líneas $B$ y pueden presentar broncograma aéreo o líquido. Son un dato ecográfico de alto riesgo de complicación. Si aparecen en zonas anteriores son signo de mal pronóstico. ${ }^{3}$

Si se observan consolidaciones subpleurales, implicarán una neumonía con riesgo de complicación, es por ello que es importante la correlación de la clínica y ecografía.

Los hallazgos ecográficos normalmente se visualizan de forma precoz, mucho antes que los estudios radiológicos convencionales. Se debe tener en cuenta que la ecografía es mucho más sensible que la radiografía; y posiblemente se diagnostique a muchos pacientes, especialmente jóvenes con cuadros leves/ moderados, que pasarían como falsos negativos en la radiografía de tórax. Precisamente por este motivo es especialmente útil en atención primaria en la que un porcentaje importante de los pacientes no tienen criterios de ingreso o de gravedad, por lo que habrá que valorar consecuentemente los hallazgos (por ejemplo: paciente clínicamente bien sin criterios de alarma, pero con consolidaciones subpleurales, que son un dato ecográfico de gravedad que probablemente requiera hospitalizarse).

Los patrones ultrasonográficos de lesiones en COVID-19 de acuerdo con la correlación clínica son: ${ }^{4}$

1. Leve: presencia de líneas $B$ de 7 milímetros de separación entre ellas o bien patrón $B 1$. Ausencia de consolidaciones o líneas $B$ de 3 milímetros de separación, ausencia de líneas $B$ que confluyen. Línea pleural normal o ligeramente engrosada.

2. Moderada: líneas $B$ de 3 milímetros de separación entre ellas o patrón B2, consolidaciones subpleurales/parenquimatosas pequeñas, irregularidades leves de pleura.

3. Grave: abundantes líneas B de 3 milímetros de separación entre ellas o patrón $B 2$, consolidaciones subpleurales generalizadas y/o consolidaciones grandes a lobares.

Con base en la clasificación anterior, el patrón B1 se refiere a la presencia de más de dos líneas $B$ bien espaciadas, difusas en las distintas áreas del tórax, denominadas septal rockets, y el patrón B2 son líneas B confluentes separadas entre ellas por $<3 \mathrm{~mm}$ (groundglass rockets) debido a la pérdida más severa de pulmón aireado.

\section{MATERIAL Y MÉTODOS}

\section{Técnica de ultrasonido}

En el Hospital General de Ecatepec Las Américas se usó un ultrasonido VINNO 5 con dos tipos de transductores: lineal (7-15 MHz) y convexo (frecuencia de 2 a $5 \mathrm{MHz}$ ); con ambos se identificaron las consolidaciones subpleurales y una vez identificadas se procedió a la medición del tamaño tanto en modo bidimensional como en modo $\mathrm{M}$.

\section{Técnica de exploración}

Previa preparación del equipo, elección de los transductores y adecuada colocación del equipo de protección (colocación de traje Tyvek, botas, guantes, gafas protectoras, N95 y careta) se procedió a insonar al paciente en posición decúbito supino tanto en ventilación espontánea como en aquellos con ventilación mecánica asistida. Se dividió cada hemitórax en cuatro cuadrantes delimitados por la línea paraesternal media, axilar anterior y axilar posterior, interceptados por una línea horizontal que pase a nivel de la línea mamaria; se comienza explorando el hemitórax anterior derecho y luego el izquierdo, en sentido cráneo caudal (comenzar la exploración a nivel de la clavícula y finalizar cuando se vea la víscera abdominal), recorriendo longitudinalmente todo el espacio intercostal entre la línea paraesternal y axilar anterior, explorar la zona lateral de la misma manera en sentido cráneo caudal poniendo especial

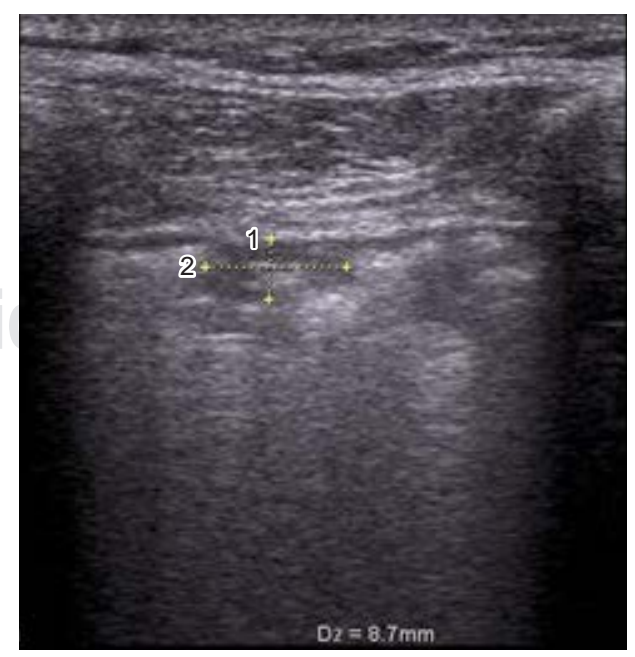

Figura 1: Consolidación subpleural en modo bidimensional con transductor lineal. 


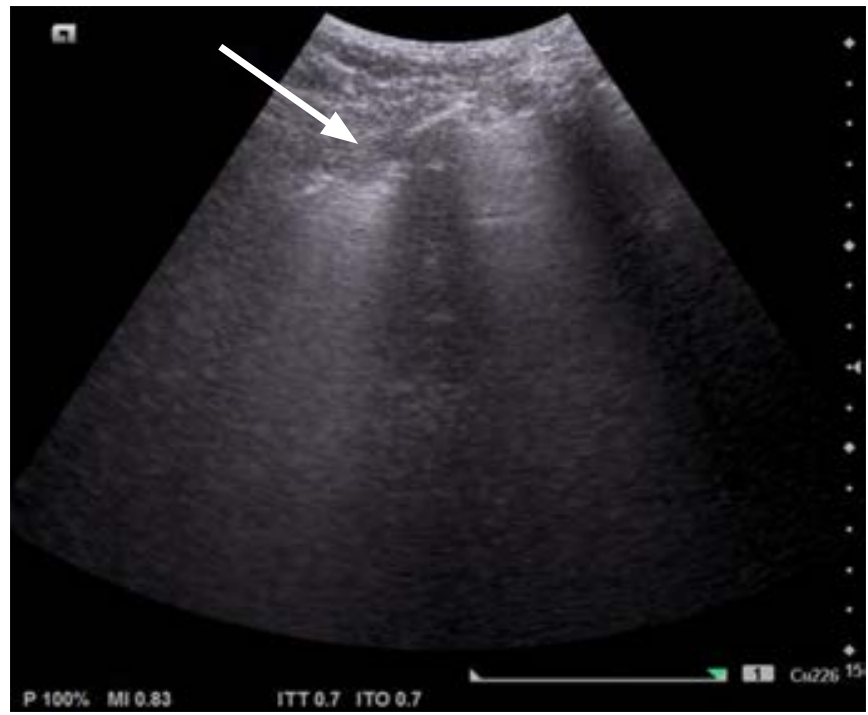

Figura 2: Consolidación subpleural (punta de la flecha) en modo bidimensional con transductor convexo.

atención en la zona axilar y finalizando en el seno costo diafragmático (lugar más frecuente de consolidaciones iniciales). El foco se situará a nivel de la línea pleural, se podrán realizar cortes longitudinales y transversales.

Posterior a la identificación de la consolidación subpleural en modo B (Figuras 1 y 2 ) se realizará la medida del tamaño de ésta en modo $\mathrm{M}$, colocando el marcador al centro de la consolidación y se procederá a evaluar el movimiento respiratorio cíclico del pulmón y posteriormente se congelará la imagen al observar por lo menos dos movimientos cíclicos y se procederá a la medición al final de la espiración, en sus medidas longitudinal y transversal, colocando el cursor para su medida en $\mathrm{mm}$ (Figuras 3 y 4 ).

\section{RESULTADOS}

Las consolidaciones subpleurales se identificaron como lesiones hipoecoicas localizadas en la región subpleural, para ser más específicos por debajo de la pleura, o bien descritas como "signo de la pleura cortada», las cuales presentan cambios en su tamaño a la inspiración y espiración.

En los pacientes en los que se realizó ultrasonido pulmonar tanto en modo $\mathrm{B}$ como en modo $\mathrm{M}$ se obtuvieron diferentes imágenes y se logró medir el tamaño de la consolidación, obteniendo una mejor calidad y tamaño en las imágenes en modo $\mathrm{M}$, principalmente en aquellos pacientes en los que se utilizó un transductor convexo. Todos los pacientes en los que se identificaron consolidaciones subpleurales de mayor tamaño cursaban con cuadros graves de la enfermedad, como síndrome de distrés respiratorio severo y aquellos que no se encontraban intubados, pero en los que se identi- ficaron consolidaciones subpleurales, tuvieron mal pronóstico y evolucionaron a deterioro y finalmente requirieron manejo avanzado de la vía aérea.

\section{DISCUSIÓN}

El ultrasonido pulmonar se ha convertido en una valiosa herramienta para el manejo de los pacientes afectados por COVID-19, no sólo a nivel hospitalario, sino también en atención primaria, tanto para el triaje (junto a la pulsioximetría) como para la toma de decisiones terapéuticas y valoración de la evolución. La auscultación no aporta mucho para el riesgo que supone al profesional, la radiología no detecta las condensaciones subpleurales que son pequeñas y la TAC en esta situación de colapso sanitario es casi inaccesible. ${ }^{5}$

La mayoría de las unidades de primer contacto como urgencias-choque, unidad de cuidados intensivos, anestesiología, etcétera, no cuentan con el transductor lineal que sería el ideal para medir e identificar las consolidaciones subpleurales; sin embargo, la mayoría sí cuenta con el transductor convexo, ya que en todos los hospitales se cuenta con el servicio de
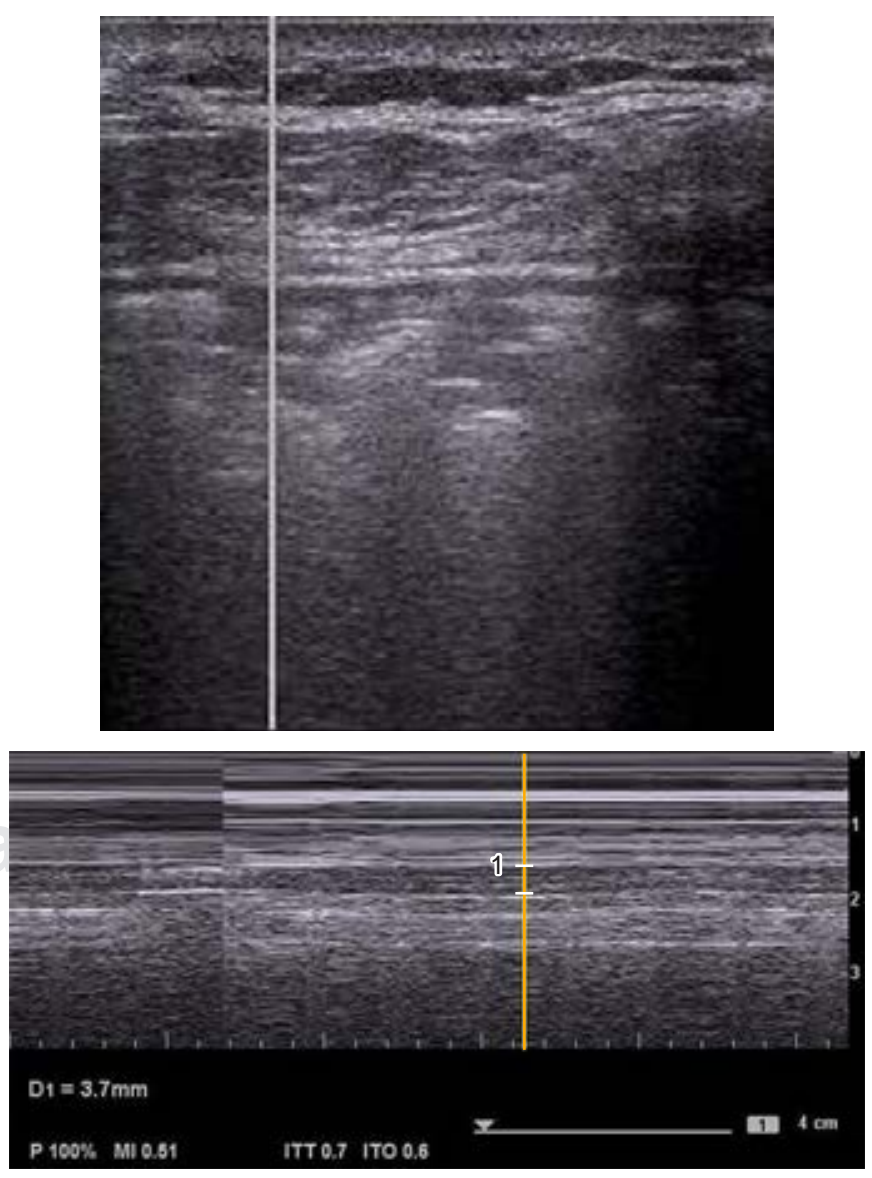

Figura 3: Consolidación subpleural en modo M con transductor lineal, medición de tamaño durante la espiración. 

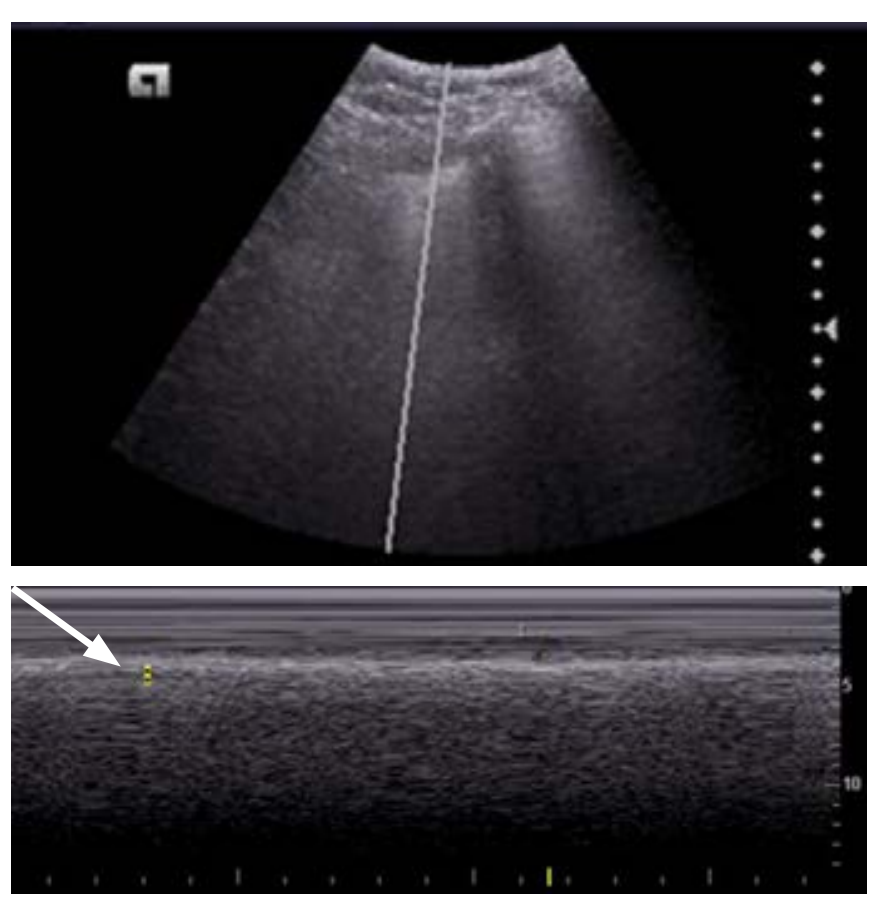

Figura 4: Consolidación subpleural (punta de la flecha) en modo M con transductor convexo.

ginecología, lo que hace más factible contar con este tipo de transductores.

Debido a que en modo $B$ con el transductor convexo es difícil medir y poder ser más objetivo en la magnitud de estas consolidaciones, propusimos la medición de dichas consolidaciones con un transductor convexo y en modo M.

\section{CONCLUSIONES}

La técnica que proponemos es medir dichas consolidaciones principalmente en modo $M$, ya que en modo $B$ no se pueden identificar claramente los bordes de la consolidación, lo cual dificulta la medición durante la inspiración y espiración, siendo este último el valor que se tomará en cuenta, como se observa en las imágenes.
Al proponer esta nueva técnica de ultrasonido se amplía el panorama acerca de la identificación, diagnóstico y monitorización de las consolidaciones subpleurales en pacientes con COVID-19, lo cual se convierte en una herramienta fácil, de bajo costo y rápida que nos permite la toma de decisiones de manera completa, además de inferir en el tratamiento y pronóstico de dichos pacientes, abriendo paso a futuros protocolos de estudio.

\section{REFERENCIAS}

1. Lichtenstein D. Novel approaches to ultrasonography of the lung and pleural space: where are we now? Breathe. 2017;13:100111.

2. González-Castro A, Escudero-Acha P, Peñasco Y, Leizaola O, Martínez de Pinillos Sánchez V, García de Lorenzo A. Cuidados intensivos durante la epidemia de coronavirus 2019. Med Intesiv. [Internet]. 2020 Marzo [citado 2020 Oct 13]44(6):351-362. doi: https://doi.org/10.1016/j.medin.2020.03.001

3. Zalaquett E, Lomoro P, Natalizi A. Ultrasonido pulmonar en COVID-19. Rev Chil Radiol. [Internet]. 2020 Junio [citado 2020 Oct 13]26(2):46-51. Disponible en: https:// scielo.conicyt.cl/scielo.php?script=sci_arttext\&pid=S071793082020000200046\&lng=es. http://dx.doi.org/10.4067/S071793082020000200046

4. Peng QY, Wang XT, Zhang LN, Chinese Critical Care Ultrasound Study Group (CCUSG). Finding of lung ultrasonography of novel coronavirus pneumonia during the 2019-2020 epidemic. Intensive Care Med. 2020. doi: 10.1007/s00134-020-05996-6

5. Villén T, Cheyayeb J, Campo R. Ecografía pulmonar para el manejo de la infección por COVID-19. Directos de SEMES. 26 abril 2020.

Conflicto de intereses: Los autores declaran no tener conflicto de intereses en relación con el presente artículo. Contribución de los autores: Todos los autores han confirmado su autoría en el documento de responsabilidades del autor, acuerdo de publicación y cesión de derechos a la Revista de Medicina Crítica.

Financiación: Los autores declaran la no existencia de financiación en relación al presente artículo.

Correspondencia:

Dra. Karen Itzel González Martínez

E-mail: karengm_89@hotmail.com 